\title{
German Medical Science als Open-Access-Publikationssystem
}

\author{
Ulrich Korwitz \\ Deutsche Zentralbibliothek für Medizin \\ Korwitz@zbmed.de
}

German Medical Science (GMS) wurde als Open-Access-Portal (Golden Road) von der Arbeitsgemeinschaft der Wissenschaftlichen Medizinischen Fachgesellschaften (AWMF), dem DIMDI und der ZB MED Köln im Jahre 2003 gegründet. Neben einer interdisziplinären Zeitschrift werden mittlerweile zwölf Fachzeitschriften und eine Vielzahl von Kongressen und Reports publiziert. Die Zahl der Zugriffe steigt stetig und liegt heute bei über 500.000 im Monat. Die Autoren behalten ihr Urheber- und Verbreitungsrecht. Zusätzlich werden die Artikel mit einer Creative-Commons-Lizenz versehen. Alle Publikationen werden begutachtet (Peer Reviewing); die Ablehnungsquote liegt bei bis zu 70 \%. Nach anfänglicher Förderung durch DFG und Bundesgesundheitsministerium wird GMS nunmehr von ZB MED und DIMDI finanziert. Das zukünftige Geschäftsmodell sieht die Erhebung von Beiträgen der Fachgesellschaften und Autoren sowie der Kongressveranstalter vor. Die Abwicklung geschieht über eine gGmbH, die von der AWMF gegründet wurde. Maßgebliche Gründe für den Erfolg von GMS sind das erfolgreiche Marketing bei den Fachgesellschaften, die hohe Qualität der Dienstleistung und die Zuverlässigkeit von Technik und redaktioneller Betreuung.

German Medical Science (GMS) was established in 2003 by the Association of German Scientific Medical Societies (AWMF), DIMDI (German Institute of Medical Documentation and Information), and the ZB MED Köln (German National Library of Medicine in Cologne) as an open access portal (i. e. the golden road). Along with an interdisciplinary journal, GMS now publishes 12 specialist journals and a wide number of congress proceedings and reports. The number of monthly visits is growing steadily and currently amounts to over 500,000 per month. At this portal the authors retain their copyright and dissemination rights. Additionally, the articles are provided with a Creative Commons license. All journals are peer-reviewed and the rejection rate is in some cases as high as $70 \%$. Subsequent to the start-up funding by the German Research Foundation (DFG) and the Federal Ministry of Health, GMS is now financed by the ZB MED and DIMDI. Its future business plan envisions charging contributions from professional associations and authors as well as congress organizers. This fee collection will be conducted through a non-profit company set up by the AWMF. The leading reasons for the success of GMS are a successful marketing strategy within the professional associations, the high quality of service, the reliability of its technical infrastructure and sound editorial support.

Die Initiative zur Gründung des Open-Access-Portals „German Medical Science (GMS)“ ging im Jahre 1999 maßgeblich von den Mitgliedern der Arbeitsgemeinschaft Wissenschaftlicher Medizinischer Fachgesellschaften (AWMF) aus. In der AWMF sind 150 wissenschaftliche Fachgesellschaften mit ca. 150.000 Mitgliedern aus allen Bereichen der Medizin zusammengeschlossen. Eine größere Zahl der Fachgesellschaften war Herausgeber von zum Teil seit vielen Jahren in kommerziellen Verlagen erscheinenden Zeitschriften. Es wurde vielfach Klage über die Konditionen der Herausgeberverträge mit den Verlagen geführt, durch die hohe Zahlungen für die Publikationsorgane zu erfolgen hatten. In den meisten Fällen blieb noch nicht einmal das Recht am Titel der Fachzeitschrift im Besitz der Fachgesellschaft. Auch die Verwertungsrechte an den Artikeln wurden an die Verlage abgetreten. Andere Fachgesellschaften wiederum monierten, dass es ihnen nicht gelungen war, einen kommerziellen Verlag zur Publikation eines Fachorgans zu motivieren. Die Verlage hatten argumentiert, dass sich eine Publikation „nicht rechnen“ würde. Gleichzeitig gab es Anfragen von Fachgesellschaften zur elektronischen Publikation der Abstracts und Proceedings ihrer Kongresse. 
In dieser Situation und bei erstarkender Open-Access-Bewegung konsultierte der Präsident der AWMF, der zugleich Vorsitzender des Beirats der Deutschen Zentralbibliothek für Medizin (ZB MED) war, diese und das mit der ZB MED kooperierende Deutsche Institut für Medizinische Dokumentation und Information (DIMDI). Die drei Partner kamen überein, die interdisziplinäre Fachzeitschrift „German Medical Science“ zu gründen und diese nach den Prinzipien des Open Access im WWW zu publizieren. Bei der Delegiertenversammlung der AWMF wurde das Projekt vorgestellt und fand breite Zustimmung.

Im Dezember 2002 wurde eine Kooperationsvereinbarung zwischen den Partnern AWMF, DIMDI und ZB MED geschlossen. Zum 1. 7. 2003 wurde das Portal „www.egms.de“ im WWW eröffnet und die ersten Artikel der Zeitschrift „German Medical Science“ erschienen online.

\section{Die Partner und ihre Rollen}

Die AWMF stellt den wissenschaftlichen Beirat (Scientific Committee) und das Editorial Board von GMS und organisiert das Peer-Review-Verfahren der Hauptzeitschrift sowie - in den einzelnen Fachgesellschaften - der Fachzeitschriften. Die 2005 gegründete GMS gGmbH ist der Vertragspartner von DIMDI und ZB MED einerseits sowie der Fachgesellschaften als Herausgeber von Fachzeitschriften und Kongressen andererseits. Dem DIMDI obliegt die Verantwortung für die technische Infrastruktur des Portals und des Publikationssystems. Dies schließt neben der technischen Implementierung die Standardisierung des Workflows, den Betrieb sowie die Langfristarchivierung der Inhalte ein. Die ZB MED hat die Projektleitung von GMS inne, betreibt aktives Marketing und stellt alle redaktionellen Dienstleistungen bereit. Im Rahmen der beiden von der DFG finanzierten Projektstufen wurde ein valides Geschäftsmodell entwickelt, das nun umgesetzt wird.

\section{Korwitz: German Medical Science als Open-Access-Publikationssystem $\quad$ S. 259 ZfBB 54 (2007) 4-5, S.258-261.}

\section{Rechtliche Rahmenbedingungen}

Grundprinzip ist der kostenfreie Zugang zu den Publikationen, wobei die Autoren das Urheberrecht und die Verbreitungsrechte ihrer Artikel im vollen Umfang behalten. Das Recht zur elektronischen Speicherung und Langzeitarchivierung wird GMS übertragen. Die Autoren können ihren Artikel sekundär an beliebiger Stelle publizieren, müssen nur auf die Primärveröffentlichung unter GMS hinweisen.

Zukünftig werden die Artikel zusätzlich unter die Creative-Commons-Lizenz (siehe http://de.creative commons.org/) gestellt, wobei eine gewerbliche Nutzung wie eine Veränderung der Texte nicht gestattet werden soll.

\section{Aufbau, Funktionalitäten und Dienste des Portals}

Die Einstiegsseite weist folgende Publikationsbereiche auf:

- Hauptzeitschrift „German Medical Science“ für hochrangige interdisziplinäre Original- und Übersichtsarbeiten aus dem Gesamtspektrum der Medizin

- Fachzeitschriften der medizinischen Fachgesellschaften, der AWMF und der Arbeitsgemeinschaft für Medizinisches Bibliothekswesen (AGMB)

- Konferenzen (Abstracts von Kongressen, z. T. erweitert, und Berichte über Kongresse)

- Forschungsberichte.

Die eingereichten Artikel der Hauptzeitschrift werden, vom Editor-in-Chief gesteuert, im anonymen Peer- Review-Verfahren begutachtet, wobei die Ablehnungsquote bei $70 \%$ liegt; bei den Fachzeitschriften wie den Kongressen obliegt die Begutachtungssteuerung den einzelnen Herausgebern. Die durchschnittliche Frist zwischen Einreichung und Annahme der (überarbeiteten) Publikation liegt bei 6-10 Wochen. 


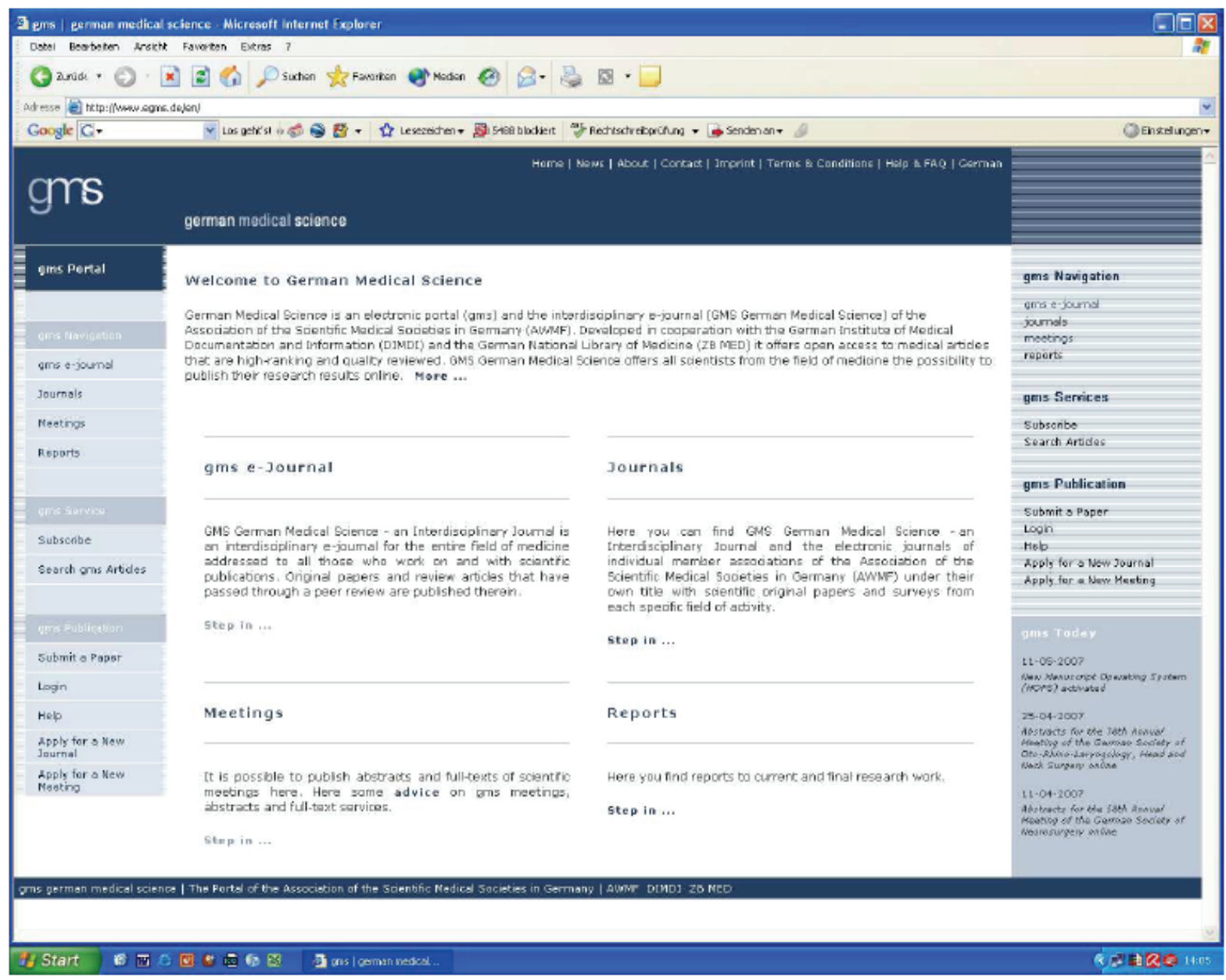

Bis Ende April 2007 wurden 1.149 Artikel in der Haupt- und den zwölf Fachzeitschriften sowie 14.552 Abstracts von 45 Kongressen veröffentlicht. Die Zugriffsfrequenz liegt bei bis zu $480.000 \mathrm{im}$ Monat.

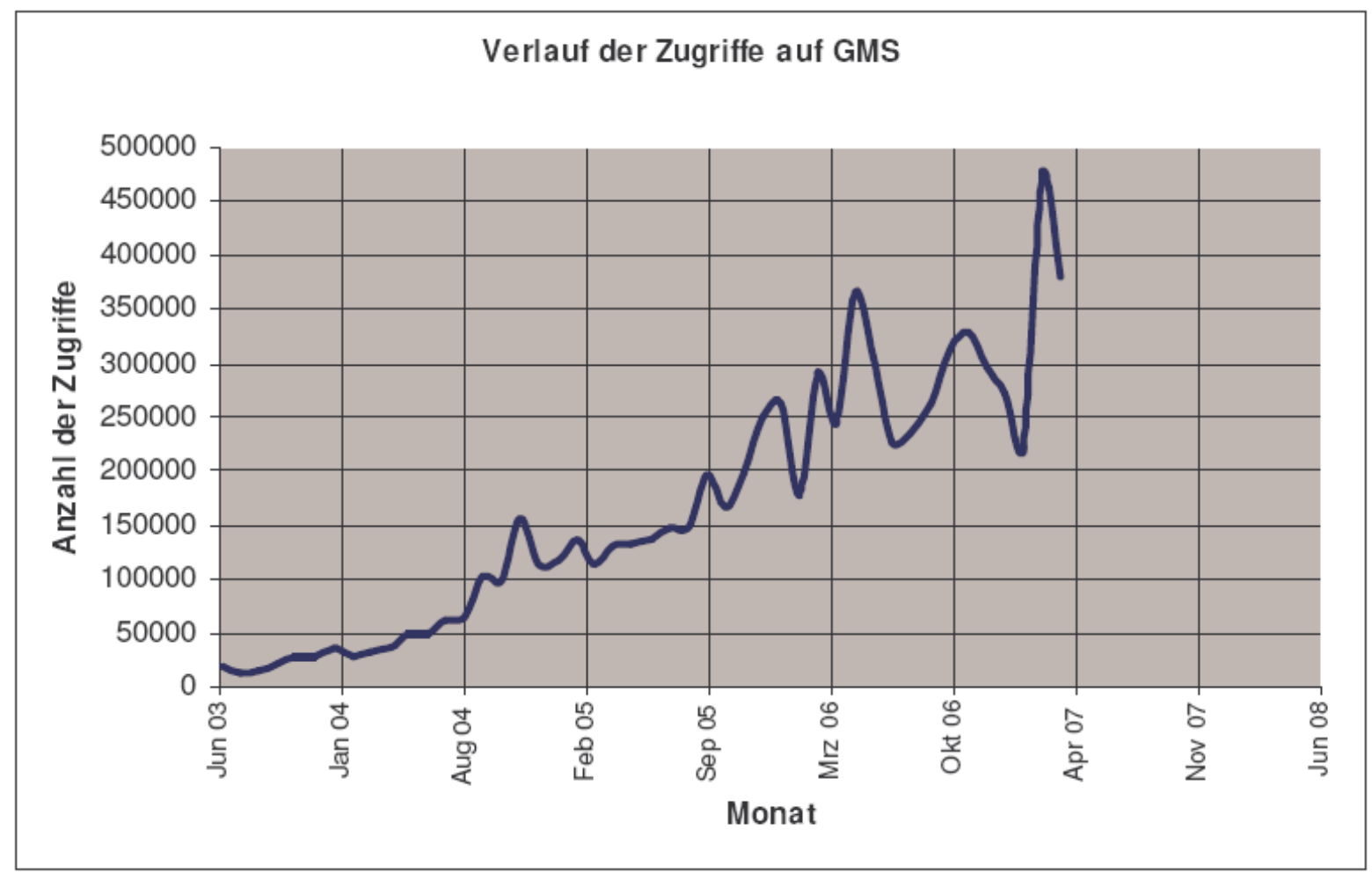


Die Publikationen sind im Volltext recherchierbar, die Metadaten im Dublin-Core-Metadatenformat werden in Datenbanken des DIMDI sowie der ZB MED (CCMed und MEDPILOT.DE) zur Recherche zur Verfügung gestellt. Eine Sacherschließung mit den Medical Subject Headings (MeSH) erfolgt für Artikel der Hauptzeitschrift. Alle Zeitschriften sind in der ZDB, der EZB und dem Directory of Open Access Journals (DOAJ) (www. doaj.org) verzeichnet. Google weist die Artikel in seinem Angebot komplett nach.

\section{Die GMS-Software im Workflow}

Es handelt sich um ein modulares System mit grundsätzlicher Trennung in Manuscript Operating System (MOPS), Publikationssystem (PUBS, beim DIMDI), Composer und zusätzlichen Werkzeugen wie Submit-Tool, Online-Review etc. bei Verwendung modernster Architektur (browserbasiert, Java, XML, ASP-fähig) und voller OAI- und OPUS-Compliance.

\section{Systemkomponenten GMS}

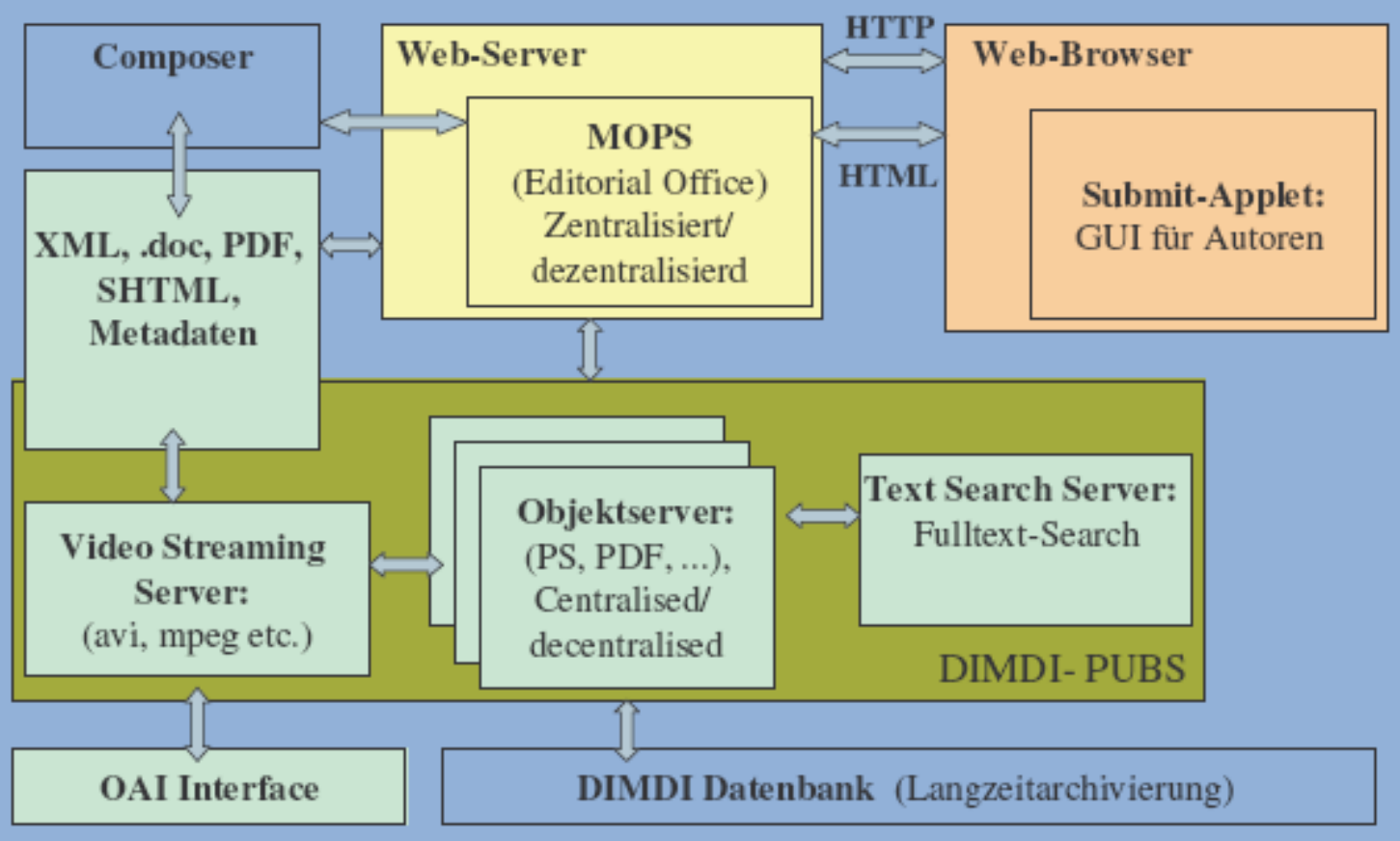

\section{Marketingmaßnahmen}

Für den Erfolg des Portals ist der enge Kundenkontakt unabdingbar. Potenziell interessierte Fachgesellschaften werden persönlich angesprochen, Publikationsbe-

$$
\begin{array}{cc}
\text { Korwitz: German Medical Science als Open-Access-Publikationssystem } & \text { S. } 260 \\
\text { ZfBB } 54 \text { (2007) 4-5, S.258-261. } &
\end{array}
$$

dürfnisse festgestellt und Anforderungen abgestimmt. Ein zeitlicher Vorlauf von drei bis sechs Monaten bei Kongressen ist notwendig, eine hohe Flexibilität auf Seiten von ZB MED und DIMDI unabdingbar.

Die vertiefte Kommunikation findet auf den halbjährlichen Treffen des Editorial Boards, den halbjährlichen Delegiertenversammlungen der AWMF sowie auf Herausgeberversammlungen statt. Auf den Tagungen des Medizinischen Fakultätentags wird regelmäßig über GMS berichtet. 


\section{Das Geschäftsmodell}

Nach Beendigung der Förderphasen durch die Deutsche Forschungsgemeinschaft und das Bundesministerium für Gesundheit ist das in der Förderzeit erarbeitete Geschäftsmodell sukzessive umzusetzen. GMS bietet den Fachgesellschaften zukünftig folgende Servicestufen an:

Basis: Peer-Review sowie formale Redaktion und technische Erfassung der Manuskripte werden von der Fachgesellschaft übernommen. Die Qualitätssicherung erfolgt in der GMS-Redaktion.

Service: Die Fachgesellschaft übernimmt das Peer-Review, die GMS-Redaktion die formale Redaktion und technische Erfassung der Beiträge.

Premium: Die GMS-Redaktion übernimmt zusätzlich das Management des Peer-Review-Prozesses und das Korrekturlesen.

Die Aufwendungen sind von den Fachgesellschaften zu erstatten, wobei neben einem Grundbetrag für die Einrichtung der Website einer neuen Zeitschrift oder eines Kongresses Kosten für jeden publizierten Artikel und Abstract berechnet werden. Die Kosten belaufen sich beispielsweise für Zeitschriftenartikel beim Basispaket auf ca. $70 €$ pro Artikel und bei Kongressen auf ca. $3 €$ pro Abstract. DIMDI und ZB MED betrachten es als ihre genuine Aufgabe, die Servicekapazitäten für die Weiterführung des GMS-Portals bereitzustellen. Die Kosten für die Publikation der Hauptzeitschrift werden komplett von AWMF, DIMDI und ZB MED getragen.

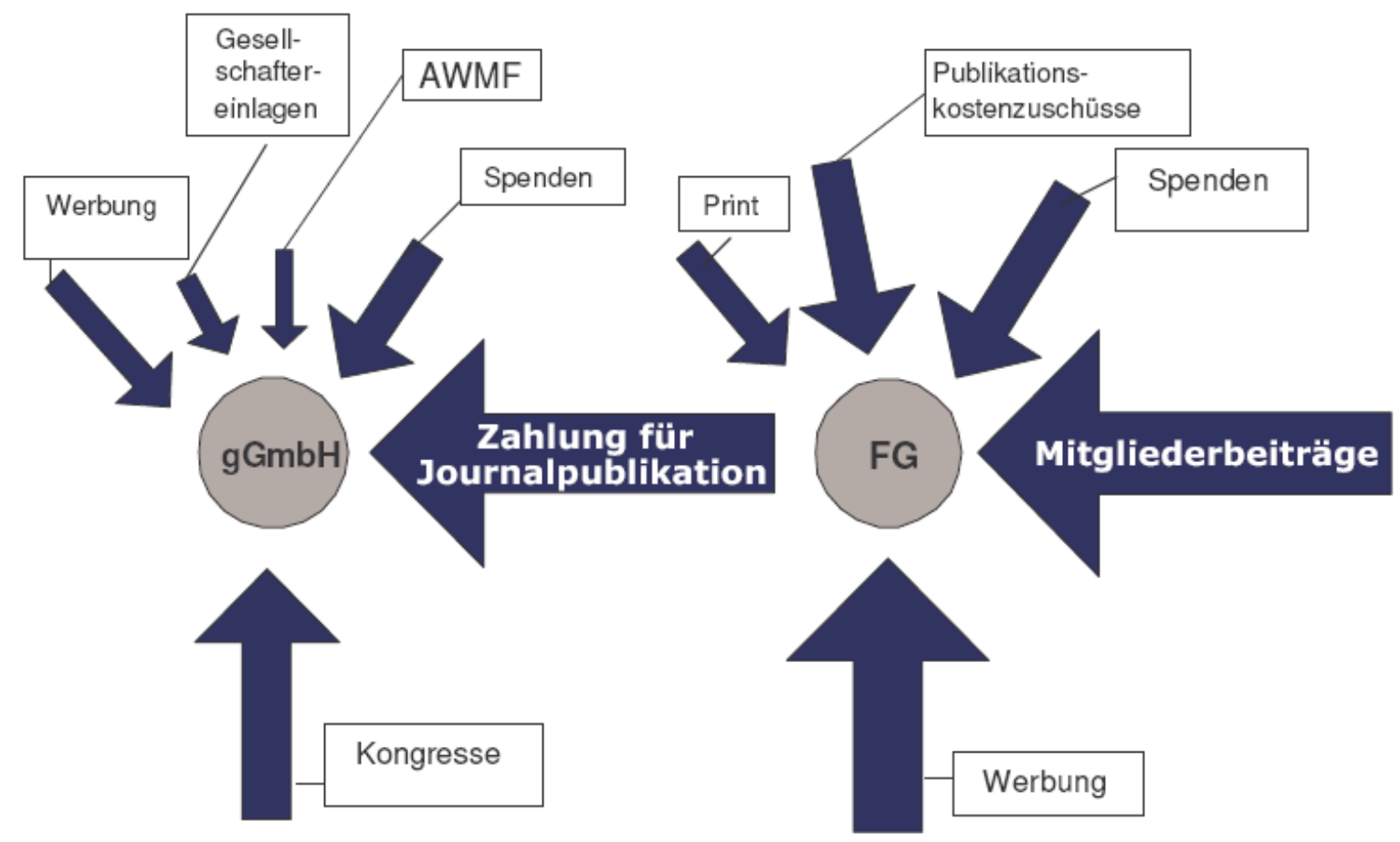

Die Finanzabwicklung erfolgt über die GMS gGmbH: die Fachgesellschaft schließt einen Vertrag mit der GMS gGmbH über die Publikation einer Zeitschrift ab, wobei sie ihre Kosten entweder aus eigenen Mitteln (Mitgliedsbeiträge, Werbeeinnahmen, Spenden) bestreitet oder von den Autoren erstattet bekommt. Diese wiederum können Publikationszuschüsse von Forschungsförderorganisationen nutzen. Gleiches gilt sinngemäß für Kongressveranstalter, wobei diese die Kosten für die Publikation der Abstracts problemlos aus den Teilnahmebeiträgen der Kongressteilnehmer bestreiten können. Die GMS gGmbH schließt wiederum Serviceverträge mit dem DIMDI und der ZB MED ab. Sonderleistungen wie „Print on demand“ werden ermöglicht. Wenn Fachgesellschaften Gesellschafter der gGmbH werden, so ermäßigen sich die zu zahlenden Kosten. Diese Möglichkeit haben bereits vier Fachgesellschaften genutzt. 


\section{Zusammenfassung}

GMS als Open-Access-Publikationsportal ist ein pragmatischer Ansatz, der in enger Kooperation mit den medizinischen Fachgesellschaften, den Kunden von GMS, entwickelt und betrieben wird. Charakteristika sind ein strenges Peer-Reviewing, die Aufrechterhaltung hoher Publikationsqualität und die Zuverlässigkeit von Redaktion und Technik bei hoher Schnelligkeit des Publikationsprozesses. Die hohe Nutzung und Zufriedenheit beweisen, dass ein Open-Access-Portal im Sinne des ,goldenen Weges" ohne hohe Aufwendun-

Korwitz: German Medical Science als Open-Access-Publikationssystem

$$
\text { ZfBB } 54 \text { (2007) 4-5, S.258-261. }
$$

gen und Kosten für die Kunden aufrechterhalten werden kann. Die erhöhte Zitierhäufigkeit der publizierten Inhalte führt zu einer Autorenzufriedenheit und dient den Interessen der Scientific Community. Die Publikationskosten werden von den Fachgesellschaften oder Kongressveranstaltern aufgebracht. Von hoher Bedeutung ist die Nähe zu den Kunden durch intensives Marketing bei fortlaufender Akquisition von neuen Inhalten. 